\title{
Minimum variance filters and mixed spectrum estimation
}

\author{
M. Durnerin, N. Martin* \\ LIS - Laboratoire des Images et des Signaux, UMR No. 5038, BP 46 - 38402 Saint Martin d'Hères Cedex, France \\ Received 19 July 1999; received in revised form 15 May 2000
}

\begin{abstract}
This paper presents a spectral density estimator based on a normalized minimum variance (MV) estimator as the one proposed by Lagunas. With an equivalent frequency resolution, this new estimator preserves the amplitude estimation lost in Lagunas one. This proposition comes from a theoretical study of MV filters that highlights this amplitude lost. Two signal types are taken into account: periodic deterministic signals (narrow-band spectral structures) and stationary random signals (broad-band spectral structures). Without selecting a smoothing window, the proposed estimator is an alternative to Fourier-based estimator and, without modeling the signal, it is a concurrent to high-resolution estimators. (C) 2000 Elsevier Science B.V. All rights reserved.
\end{abstract}

\section{Zusammenfassung}

Dieser Artikel stellt einen Schätzer für die Spektraldichte vor, der auf einem Schätzer normierter minimaler Varianz (MV), wie der von Lagunas vorgschlagene, basiert. Dieser neue Schätzer erhält die Amplitudenschätzung, die in dem Ansatz von Lagunas verlorenging, während die Frequenzauflösung gleich bleibt. Der Vorschlag stammt aus einer theoretischen Studie von MV Filtern, die den Amplitudenverlust betont. Es werden zwei Signalarten betrachtet: periodische deterministische Signale (schmalbandige spektrale Strukturen) und stationäre Zufallssignale (breitbandige spektrale Strukturen). Ohne Auswahl eines Glättungsfensters ist der vorgeschlagene Schätzer eine Alternative zu Schätzern, die auf Fourier-Methoden basieren, und ohne Signalmodellierung ein Konkurrent zu hochauflösenden Schätzern. (C) 2000 Elsevier Science B.V. All rights reserved.

\section{Résumé}

Cet article présente un estimateur de densité spectrale défini à partir d'un estimateur du Minimum de Variance (MV) Normalisé tel que celui proposé par Lagunas. Avec une résolution fréquentielle équivalente, l'objectif de ce nouvel estimateur est de préserver l'estimation de l'amplitude contrairement à l'estimateur de Lagunas. Cette proposition s'appuie sur l'étude de la fonction de transfert du filtre MV. Deux types de signaux sont considéré: des signaux déterministes périodiques (dont la structure spectrale est à bande étroite) et des signaux aléatoires stationnaires (dont la structure spectrale est à large bande). Sans avoir à choisir une fenêtre d'apodisation, l'estimateur proposé est une alternative aux estimateurs de Fourier, et, sans appliquer de modèle au signal, est un concurrent des estimateurs paramétriques. (C) 2000 Elsevier Science B.V. All rights reserved.

Keywords: Spectral analysis; Data filtering; Minimum variance method

* Corresponding author. Tel.: + 33-4-76-82-62-69; fax: + 33-4-76-82-63-84.

E-mail addresses: mathieu.durnerin@art.alcatel.fr (M. Durnerin), nadine.martin@inpg.fr (N. Martin). 


\section{Introduction}

In spectral analysis, the minimum variance (MV) method holds an important place with regard to classic Fourier-based methods. The method was first developed by Capon [2] for frequency wavenumber analysis in seismic array processing, then by Lacoss [7]. Capon's method estimates the signal power from a filter bank calculated from the signal itself. The filters are constrained in order to reject the signal power at all frequencies but the desired one. Lagunas [8] has proposed an estimation of the spectral density from Capon's power estimator by introducing the filter bandwidth.

The wide application of these methods comes from the fact that no model is applied to the signal. The filter signal dependency implies optimal properties for its design which is not the case for Fourier analysis [7]. The use of the MV estimator has been conclusive in many situations: in acoustics to characterize hydrodynamic fluids [12], in room acoustics to analyze the impulse response of concert halls [11] and in geophysics [10]. All these applications concern non-stationary signals. In this case, MV methods can be extended to non-stationary signals by mean of a gliding time window under the assumption of local stationarity [1]. Thus, the properties of these estimators are all deduced from those of the stationary case. This justifies the interest of the study presented here for finite duration signals.

Our paper copes with the analysis of filters induced by MV constraints. In particular, the designed filter nature is of interest to study the normalization proposed by Lagunas. Many statistical studies had been carried on the MV estimator $[3,5,13,15]$. Little of them have considered it as a filter adapted to the data set [6]. Due to the signal dependency, this study relies on analytical developments and also on simulated filters from finite duration signals. From these results, we explain the behavior of these estimators, mainly for the amplitude estimation, and we deduce a new MV estimator. Its principle is close to Lagunas one but a different scaling significantly improves the performance. A statistical study illustrates the preservation of the amplitude value and the increase of the frequency resolution.
Section 2 briefly recalls the MV filters design and the derivation of the normalized estimator. In Section 3, the frequency response of the MV filter is studied for a complex exponential signal embedded in an additive white noise. This filter design is analyzed according to the signal-to-noise ratio and the exponential frequency value. These filters are empirically generalized for mixed spectrum. From these results, the properties of the normalized estimator are deduced in Section 4. In Section 5, we propose a new estimator whose properties are illustrated on simulations from a mixed spectrum.

\section{Minimum variance (MV) and normalized minimum variance (NMV) methods}

\subsection{Minimum variance method (Capon's method)}

The MV estimator here presented references to the data filtering concept $[2,4,5,7]$. Let $\{x(n)\}$ be a wide-sense stationary random process sampled at $t_{\mathrm{e}}$ and $S_{x}(v)$ its power spectral density function (PSD) at frequency $v$. Let us apply $x(n)$ as a filter signal input; this filter is designed at a frequency $v_{\mathrm{F}}$ in order to estimate the signal power at this frequency. Two constraints are assigned to the filter:

(1) The frequency response $A_{v_{\mathrm{F}}}(v)$ must be equal to 1 at frequency $v_{\mathrm{F}}$ :

$A_{v_{\mathrm{F}}}\left(v_{\mathrm{F}}\right)=1$.

(2) The power out of the filter due to other frequencies than $v_{\mathrm{F}}$ must be minimized. This is equivalent to minimizing the global output power $P_{\mathrm{MV}}\left(v_{\mathrm{F}}\right)[6]$ :

$P_{\mathrm{MV}}\left(v_{\mathrm{F}}\right)=\boldsymbol{A}_{v_{\mathrm{F}}}^{\mathrm{H}} \boldsymbol{R}_{x} \boldsymbol{A}_{v_{\mathrm{F}}}$,

with $\boldsymbol{A}_{v_{\mathrm{F}}}^{\mathrm{T}}=(a(0), a(1), \ldots, a(M-1))$ as the impulse response coefficients vector at $v_{\mathrm{F}}$, and $\boldsymbol{R}_{x}$ as the $M \times M$ autocorrelation matrix of the input signal $x$. Notice that

$$
\begin{aligned}
\boldsymbol{A}_{v_{\mathrm{F}}}(v)= & a(0)+a(1) \mathrm{e}^{-2 \pi \mathrm{j} v t_{\mathrm{e}}}+\cdots \\
& +\mathrm{a}(M-1) \mathrm{e}^{-2 \pi \mathrm{j}(M-1) v t_{\mathrm{e}}} \\
= & \boldsymbol{E}_{v_{\mathrm{F}}}^{\mathrm{H}} \boldsymbol{A}_{v_{\mathrm{F}}},
\end{aligned}
$$


where

$\boldsymbol{E}_{v_{\mathrm{F}}}^{\mathrm{T}}=\left(1, \mathrm{e}^{2 \pi \mathrm{j} v_{\mathrm{F}} t_{\mathrm{e}}}, \ldots, \mathrm{e}^{2 \pi \mathrm{j}(M-1) v_{\mathrm{F}} t_{\mathrm{e}}}\right)$.

The superscripts ()$^{\mathrm{T}}$ and ()$^{\mathrm{H}}$ denote transpose and Hermitian transpose.

The minimization of (2) under the constraint (1) by Lagrange multiplier technique [3] yields the impulse response and the output power. This defines the MV filter:

$\boldsymbol{A}_{v_{\mathrm{F}}}=\frac{\boldsymbol{R}_{x}^{-1} \boldsymbol{E}_{v_{\mathrm{F}}}}{\boldsymbol{E}_{v_{\mathrm{F}}}^{\mathrm{H}} \boldsymbol{R}_{x}^{-1} \boldsymbol{E}_{v_{\mathrm{F}}}}$

and

$P_{\mathrm{MV}}\left(v_{\mathrm{F}}\right)=\frac{1}{\boldsymbol{E}_{v_{\mathrm{F}}}^{\mathrm{H}} \boldsymbol{R}_{x}^{-1} \boldsymbol{E}_{v_{\mathrm{F}}}}$.

This filter is evaluated at all frequencies $v_{\mathrm{F}}$ lying between 0 and half of Shannon frequency $v_{\mathrm{e}}=1 / t_{\mathrm{e}}$. The autocorrelation matrix $\boldsymbol{R}_{x}$ must be estimated but the choice of such an estimator is not the purpose of this paper.

This filter design assumes no hypothesis on the signal itself. No model and no a priori information are needed. In Fourier analysis, the impulse response at each frequency is equal to an exponential at this frequency. The exponential is limited in time by a window equal to the width of the analyzed signal. Capon's filter behaves as a window calculated from an estimate of the covariance matrix of the signal. This dependency upon the signal ensures better properties compared with Fourier analysis. A comparative study is discussed in [7,11].

\subsection{Links with a linear minimum variance unbiased estimator}

For the particular case of a complex exponential embedded in a complex Gaussian noise, a linear minimum variance unbiased (LMVU) estimator can be defined [6] and is closely connected to Capon's estimator. The exponential amplitude is estimated by the output of a filter whose impulse response is also defined by Eq. (4) but by setting the noise instead of the signal correlation matrix. The variance of this estimator is given by Eq. (5) by setting the noise correlation matrix too. When the signal is composed of more than one exponential, the matrix in Eqs. (4) and (5) for the LMVU estimator is the correlation matrix of the noise plus all the exponentials which are not at the frequency of the filter. To do that, the exponential frequency and the noise correlation matrix need to be known. The LMVU estimator leads to a maximum likelihood estimation of the amplitude which is not the case of Capon's one. Being defined for a stationary random process and evaluated at all frequencies, Capon is more general than LMVU. The filter output power gives an estimate of the input signal power at the filter frequency whatever the signal is. As one might expect, this estimate at a defined frequency strongly depends on the signal content at other frequencies, and therefore on the noise, and on the number of filter coefficients.

\subsection{Normalized minimum variance method (Lagunas method)}

$P_{\mathrm{MV}}\left(v_{\mathrm{F}}\right)$ is homogeneous to power but not to a spectral density function since the area under the estimated function does not represent the total power of the analyzed signal. Lagunas [8] proposed a method to derive the spectral density from the MV power. Assuming that the true power density $S_{x}(v)$ is flat around $v_{\mathrm{F}}$ and is roughly equal to $S_{x}\left(v_{\mathrm{F}}\right)$, Eq. (2) can be written as

$$
\begin{aligned}
P_{\mathrm{MV}}\left(v_{\mathrm{F}}\right) & =\int_{-v_{\mathrm{e}} / 2}^{+v_{\mathrm{e}} / 2}\left|A_{v_{\mathrm{F}}}(v)\right|^{2} S_{X}(v) \mathrm{d} v \\
& \approx S_{X}\left(v_{\mathrm{F}}\right) \int_{-v_{\mathrm{e}} / 2}^{+v_{\mathrm{e}} / 2}\left|A_{v_{\mathrm{F}}}(v)\right|^{2} \mathrm{~d} v .
\end{aligned}
$$

Note that this formula assumes that the filter is a narrow bandpass one. So the MV filter output power is linked to the spectral density of the input signal by a factor which is the noise-equivalent bandwidth $B_{\mathrm{e}}$ of the filter owing to the MV constraint equation (1). Using Parseval relation, this factor is written as

$$
\int_{-v_{\mathrm{e}} / 2}^{+v_{\mathrm{e}} / 2}\left|A_{v_{\mathrm{F}}}(v)\right|^{2} \mathrm{~d} v=\frac{1}{t_{\mathrm{e}}} \boldsymbol{A}_{v_{\mathrm{F}}}^{\mathrm{H}} \boldsymbol{A}_{v_{\mathrm{F}}}=B_{\mathrm{e}} .
$$


Combining (4)-(7) the NMV PSD estimator $S_{\mathrm{NMV}}\left(v_{\mathrm{F}}\right)$ is finally written as

$S_{\mathrm{NMV}}\left(v_{\mathrm{F}}\right)=t_{\mathrm{e}} \frac{\boldsymbol{E}_{v_{\mathrm{F}}}^{\mathrm{H}} \boldsymbol{R}_{x}^{-1} \boldsymbol{E}_{v_{\mathrm{F}}}}{\boldsymbol{E}_{v_{\mathrm{F}}}^{\mathrm{H}} \boldsymbol{R}_{x}^{-2} \boldsymbol{E}_{v_{\mathrm{F}}}}$.

\section{Analysis of the MV filter}

An MV estimator study was already performed in [14]. It shows how the estimation converges to the true spectrum with regard to the filter order $M$ and the noise level. Our aim here is to point out specific properties of the MV filter for explaining the NMN estimator behavior.

The MV filter depends on the signal so that the frequency response has a different shape and then different properties according to signal characteristics. An analytical derivation of the filter should have been considered for each type of signal. We derived it only for a complex exponential embedded in white noise. Finally, we discuss the case of a simulated mixed spectrum.

\subsection{Analytical study of the $M V$ filter}

The MV filter is examined for a signal $x(n)$ which is a complex exponential of frequency $v_{\exp }$ in an additive complex white noise $w(n)$ of power $\sigma^{2}$. Let the complex amplitude of the exponential be $C e^{\mathrm{j} \Phi}$ where $\Phi$ is a uniformly distributed random phase:

$\boldsymbol{X}=C e^{\mathrm{j} \Phi} \boldsymbol{E}_{v_{\text {exp }}}+\boldsymbol{W}$

where

$\boldsymbol{X}^{\mathrm{T}}=(x(0), x(1), \ldots, x(M-1)) \quad$ and

$\boldsymbol{W}^{\mathrm{T}}=(w(0), w(1), \ldots, w(M-1))$

The autocorrelation matrix of the signal $\boldsymbol{X}$ is written as

$\boldsymbol{R}_{x}=C^{2} \boldsymbol{E}_{v_{\text {exp }}} \boldsymbol{E}_{v_{\text {exp }}}^{\mathrm{H}}+\sigma^{2} \boldsymbol{I}$,

where $\boldsymbol{I}$ is the $M \times M$ identity matrix.

Using the Sherman-Morrison formula [4], the inverse of the autocorrelation matrix turns out to be

$\boldsymbol{R}_{x}^{-1}=\frac{1}{\sigma^{2}}\left(\boldsymbol{I}-\frac{C^{2} / \sigma^{2}}{1+M C^{2} / \sigma^{2}} \boldsymbol{E}_{v_{\text {exp }}} \boldsymbol{E}_{v_{\text {exp }}}^{\mathrm{H}}\right)$.
Let $Q=\left(C^{2} / \sigma^{2}\right) /\left(1+M C^{2} / \sigma^{2}\right)$ and note that $C^{2} / \sigma^{2}$ represents the signal-to-noise ratio. Substituting Eq. (10) into Eq. (4) yields the impulse response at frequency $v_{\mathrm{F}}$ :

$\boldsymbol{A}_{v_{\mathrm{F}}}=\frac{\boldsymbol{E}_{v_{\mathrm{F}}}-Q \boldsymbol{E}_{v_{\text {exp }}} \boldsymbol{E}_{v_{\text {exp }}}^{\mathrm{H}} \boldsymbol{E}_{v_{\mathrm{F}}}}{M-Q\left|\boldsymbol{E}_{v_{\mathrm{F}}}^{\mathrm{H}} \boldsymbol{E}_{v_{\text {exp }}}\right|^{2}}$.

The filter frequency response adapted to the signal $x(n)$ at frequency $v_{\mathrm{F}}$ is then deduced:

$$
\begin{aligned}
A_{v_{\mathrm{F}}}(v) & =\boldsymbol{E}_{v}^{\mathrm{H}} \boldsymbol{A}_{v_{\mathrm{F}}}=\frac{\boldsymbol{E}_{v}^{\mathrm{H}} \boldsymbol{E}_{v_{\mathrm{F}}}-Q \boldsymbol{E}_{v}^{\mathrm{H}} \boldsymbol{E}_{v_{\text {exp }}} \boldsymbol{E}_{v_{\mathrm{exp}}}^{\mathrm{H}} \boldsymbol{E}_{v_{\mathrm{F}}}}{M-Q\left|\boldsymbol{E}_{v_{\mathrm{F}}}^{\mathrm{H}} \boldsymbol{E}_{v_{\text {exp }}}\right|^{2}} \\
& =\frac{D\left(v_{\mathrm{F}}-v\right)-Q M D\left(v_{\mathrm{exp}}-v\right) D\left(v_{\mathrm{F}}-v_{\mathrm{exp}}\right)}{1-Q M\left|D\left(v_{\mathrm{F}}-v_{\mathrm{exp}}\right)\right|^{2}},
\end{aligned}
$$

where Dirichlet kernel $D\left(v_{k}-v_{i}\right)$ is equal to

$$
\begin{aligned}
D\left(v_{k}-v_{i}\right) & =\frac{1}{M} \boldsymbol{E}_{v_{i}}^{\mathrm{H}} \boldsymbol{E}_{v_{k}}=\frac{1}{M} \sum_{l=0}^{M-1} \mathrm{e}^{2 \pi \mathrm{j}\left(v_{k}-v_{i}\right) l t_{\mathrm{e}}} \\
& =\mathrm{e}^{\pi \mathrm{j}\left(v_{k}-v_{i}\right)(M-1) t_{\mathrm{e}}} \frac{\sin \left(\pi\left(v_{k}-v_{i}\right) M t_{\mathrm{e}}\right)}{M \sin \left(\pi\left(v_{k}-v_{i}\right) t_{\mathrm{e}}\right)} .
\end{aligned}
$$

If the filter frequency $v_{\mathrm{F}}$ is equal to the exponential frequency $v_{\exp }$, Eq. (11) becomes

$\left|A_{v_{\text {exp }}}(v)\right|^{2}=D\left(v_{\exp }-v\right)^{2}$.

The exponential is filtered by a narrow-band filter centered at the exponential frequency with its maximum equal to 1 (Fig. 1b). In this particular case of one exponential in noise, MV method at the exponential frequency is reduced to Fourier estimator with a filter length fixed by the order $M$ and a -3 $\mathrm{dB}$ bandwidth equal to $1 /\left(M t_{\mathrm{e}}\right)$. We can notice that this quantity is frequently used for scaling Capon power at all frequencies even though the signal is more complex than one exponential $[5,6]$.

If the signal-to-noise ratio is small $\left(C^{2} / \sigma^{2} \ll 1\right)$, $Q$ tends to 0 and the squared modulus of the frequency response (11) is

$\left|A_{v_{\mathrm{F}}}(v)\right|^{2} \approx\left|D\left(v_{\mathrm{F}}-v\right)\right|^{2}$.

In this latter case, the filter frequency response modulus is also a Dirichlet's kernel centered at the filter frequency. This case is similar to Fourier analysis. The MV filter rejection is the same at all 
frequencies since the signal power is high at all frequencies.

If the signal-to-noise ratio is greater than $1\left(C^{2} / \sigma^{2} \gg 1\right)$, QM tends to 1 and Eq. (11) shows that the filter is no longer a Dirichlet's kernel as in Fourier analysis. In this simple case of a single exponential frequency, the structure of the frequency response clearly explains the MV principle. The denominator in (11) is only a constant whereas the numerator is the difference between two Dirichlet's kernels. The first is centered at the filter frequency $v_{\mathrm{F}}$ and the second at the exponential frequency $v_{\exp }$. The second kernel is smoothed by a factor which depends on $\left(v_{F}-v_{\text {exp }}\right)$. We developed the MV filter shape in two cases to highlight its behavior.

\subsection{Case 1: the filter frequency $v_{\mathrm{F}}$ is far from the exponential frequency $v_{\mathrm{exp}}$}

The smoothing factor $D\left(v_{\mathrm{F}}-v_{\mathrm{exp}}\right)$ tends to zero. Then, Eq. (11) is reduced to Eq. (13) when $v$ is different from $v_{\exp }$ and vanishes when $v$ tends to $v_{\text {exp }}$.

In this case, the response is a Dirichlet's kernel except at the exponential frequency where the response is minimized. This latter point makes the difference with Fourier estimator according to the MV constraint. This case is illustrated by Fig. 1c which compares the theoretical curve to a simulated one.

\subsection{Case 2: the filter frequency $v_{\mathrm{F}}$ is close to the exponential frequency $v_{\exp }$}

Given that $\left(v_{\mathrm{F}}-v_{\text {exp }}\right)$ is low, the smoothing factor $D\left(v_{\mathrm{F}}-v_{\exp }\right)$ in (11) plays a prominent part and the frequency response can have two main lobes as shown in Fig. 1d. Furthermore, as soon as $v<\min \left(v_{\mathrm{F}}, v_{\exp }\right)$ and $v>\max \left(v_{\mathrm{F}}, v_{\exp }\right),\left|A_{v_{\mathrm{F}}}(v)\right|^{2}$ is greater than 1 (cf. algebra details in Appendix A). The frequency response is not maximum at $v_{\mathrm{F}}$ but at a frequency which lays outside the frequency range $v_{\text {exp }}$ to $v_{\mathrm{F}}$. This property is shown in Fig. $1 \mathrm{~d}$. In this case, we would like to emphasize that the filter is no more a narrow-band pass filter centered at $v_{\mathrm{F}}$ and that the maximum of the frequency response can be more than unity.

\subsection{Synthesis of the MV filter's analysis}

The filter's behavior is the same whether its frequency belongs to a band where the signal power level is maximum or minimum (case 1). In the neighborhood of this filter frequency, the frequency response is that of a narrow-band filter centered at this frequency and with its maximum equal to 1 . Outside this neighborhood, the response is minimized at each frequency where the signal power level is high. This result was known, but, moreover, when the signal power is small, the frequency response can have secondary lobes with maxima much greater than one. The areas of these lobes could even be greater than the area of the lobe containing the filter frequency. Note that this situation could not appear in the analytical example, but such a behavior is encountered when the response is constraint at more than one frequency.

For illustrating this point of view, we present the analysis of a more complex signal composed of a sum of a noised sine wave and a broad-band signal simulated by a white noise filtered by a lowpass filter. The MV power estimation of this simulated signal is plotted in Fig. 2a. Fig. 2d shows the response in a frequency range where the signal power is small, Fig. 2b that for the broad-band signal and Fig. $2 \mathrm{f}$ the response at the exponential frequency.

When the filter frequency is close to a maximum of the signal power, the filter is no longer a narrow band. The constraint of unity at the filter frequency is maintained but there is no relation for constraining the filter to be maximum at this frequency. On the contrary, such a response is constrained to be minimum at a frequency close to this filter frequency. So a greater maximum should be at a frequency on the other side of that frequency. This behavior detailed in case 2 is due to the filter structure of the filter frequency which is close to an exponential frequency (Fig. 2e) or close to a broad band (Fig. 2c). The other parts of the frequency response are similar to case 1.

In conclusion, Capon's filters are not always narrow-band filters centered at the filter frequency $v_{\mathrm{F}}$. In fact, the design constraints, the unit gain at $v_{\mathrm{F}}$ and the rejection of the signal power at the other frequencies, are not the only relevant constraints to 


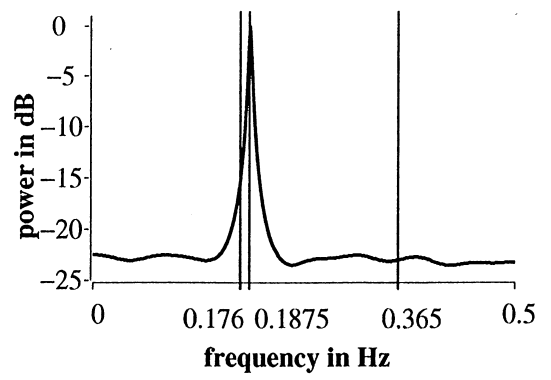

(a)

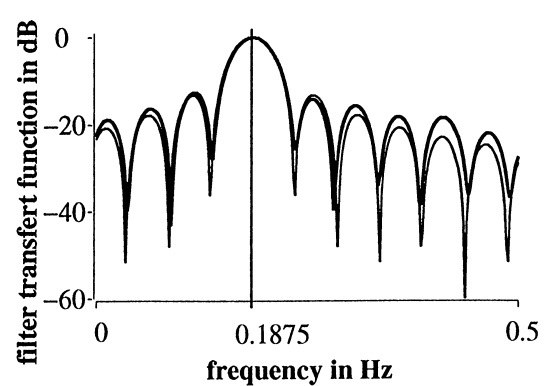

(b)

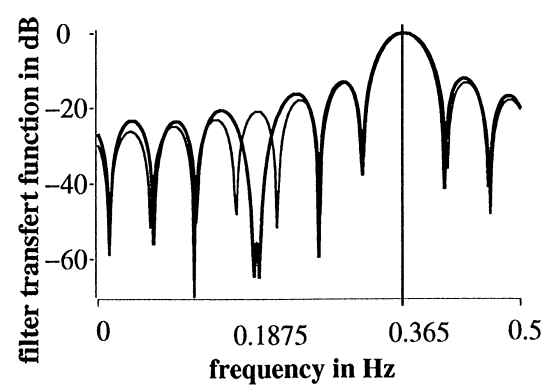

(c)

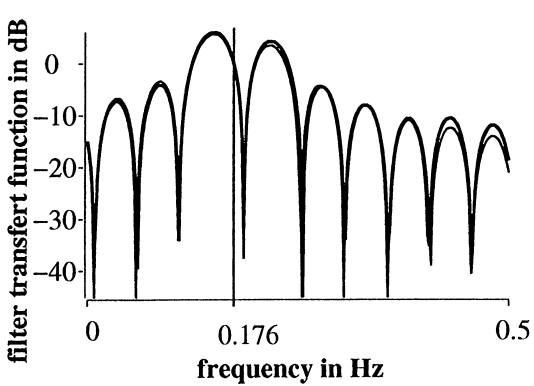

(d)

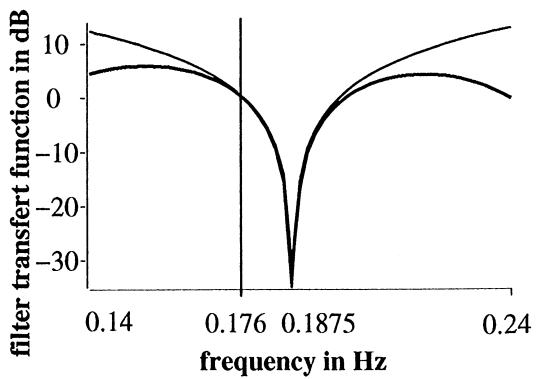

(e)

simulation

Fig. 1. Comparison of the squared magnitude of simulated MV frequency responses with our theoretical derivation at various filter frequencies $v_{\mathrm{F}}$. Signal: $v_{\exp }=0.1875 \mathrm{~Hz}$, unit amplitude, signal-to-noise ratio $10 \mathrm{~dB}$, frequency sampling $1 \mathrm{~Hz}, 1024$ points. (a) MV power estimate, order 20 , (b) $v_{\mathrm{F}}=0.1875 \mathrm{~Hz}$, at the exponential frequency, theoretical derivation given by (12), (c) $v_{\mathrm{F}}=0.365 \mathrm{~Hz}$, far from the exponential frequency, case 1 of the theoretical derivation, (d) $v_{\mathrm{F}}=0.176 \mathrm{~Hz}$, close to the exponential frequency, case 2 of the theoretical derivation, (e) $v_{\mathrm{F}}=0.176 \mathrm{~Hz}$ as in (d) but zoom in $[0.14 \mathrm{~Hz}, 0.24 \mathrm{~Hz}]$, theoretical derivation given in Appendix A.

designing a narrow-band filter. At frequencies where signal power is small, the filter is free and the frequency response can be very high. But, the output power is always representative of the signal power due to the fact that the product $|A(v)|^{2} S_{x}(v)$ is almost equal to zero whatever the value of the frequency response may be.
This particular behavior of Capon's filter was not previously pointed out. More often, the filter was explicitly or implicitly considered as a narrow-band filter [9, p.351, 6, p.370, 8]. Therefore, the normalization of the MV method by the bandwidth as defined in (7) is only actual in the case of a narrowband filter. In the other cases, the measurement of 


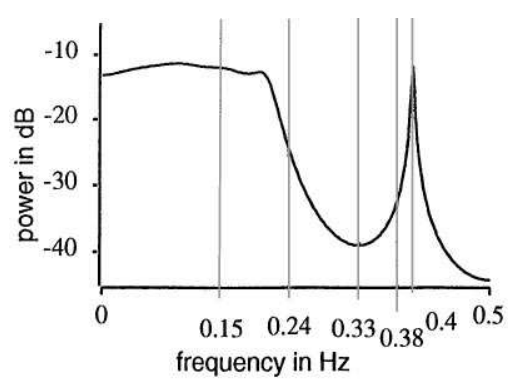

(a)

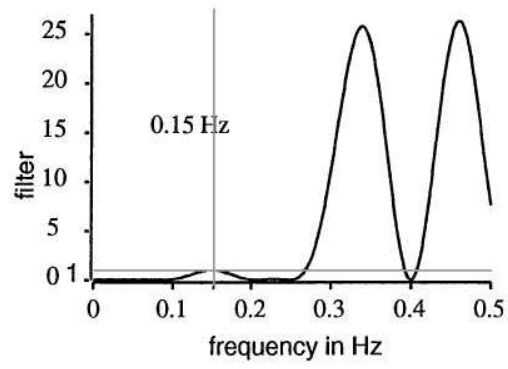

(b)

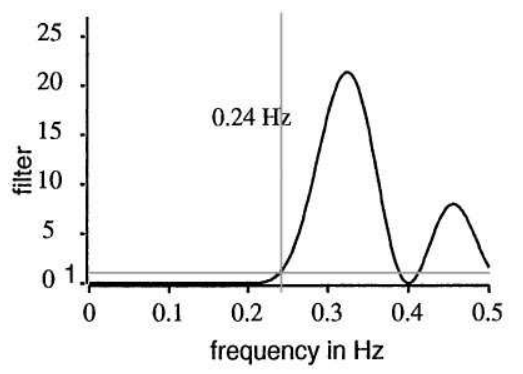

(c)

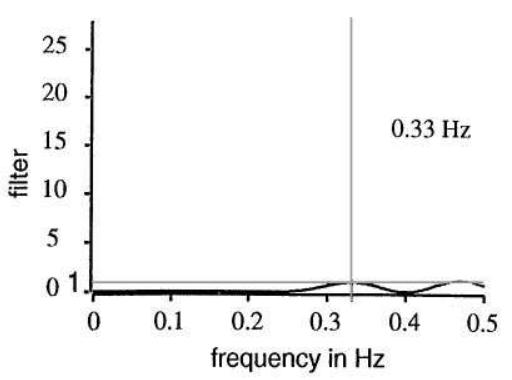

(d)

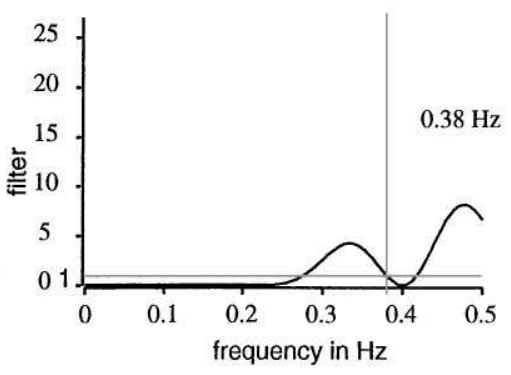

(e)

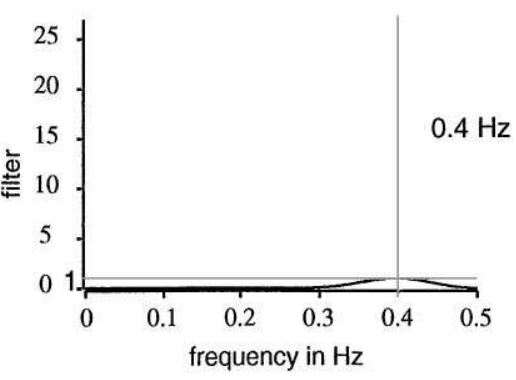

(f)

Fig. 2. Squared magnitude of simulated MV frequency responses at various filter frequencies $v_{\mathrm{F}}$. Signal: sum of a sine $(0.4 \mathrm{~Hz}$, magnitude 0.5 , signal-to-noise ratio $25 \mathrm{~dB}$ ) and of a white noise (variance 1) filtered by an elliptic low-pass filter (order 12, attenuation $80 \mathrm{~dB}$ from $0.22 \mathrm{~Hz}$ to $0.25 \mathrm{~Hz}$ ), frequency sampling $1 \mathrm{~Hz}, 256$ points. (a) MV power (order 12), (b) filter frequency $0.15 \mathrm{~Hz}$ in the broad band, (c) filter frequency $0.24 \mathrm{~Hz}$ close to the broad band, (d) filter frequency $0.33 \mathrm{~Hz}$ in the small power band, (e) filter frequency $0.38 \mathrm{~Hz}$ close to the exponential frequency, (f) filter frequency $0.4 \mathrm{~Hz}$ at the exponential frequency.

the bandwidth includes the area of huge lobes which are not significant. This conclusion explains why the noise-equivalent bandwidth cannot be considered as a measure of the frequency resolution as in Fourier analysis.

\section{Properties of NMV method}

The main property of the NMV method is a refining of power peaks. Each power variation of the
MV estimator is enhanced by unrealistic transformations. This estimation has an interest as a frequency estimator but not as an amplitude one. This spectral refinement property can be explained from the previous study by evaluating the amplitude ratio $P_{\mathrm{MV}}(v) / S_{\mathrm{NMV}}(v)$ at the exponential frequency $v_{\exp }$ and at a close frequency $\left(v_{\exp }+\varepsilon\right)$ when $\varepsilon$ tends to 0 .

According to Lagunas assumption (Section 2.3), the power $P_{\mathrm{MV}}(v)$ defined in (5) and the 
PSD $S_{\mathrm{NMV}}(v)$ defined in (8) are linked by the noiseequivalent bandwidth $B_{\mathrm{e}}$ defined in Eq. (7). When the filter frequency $v_{\mathrm{F}}$ equals the exponential frequency $v_{\text {exp }}$, the frequency response is given by Eq. (12). So the bandwidth noticed $B_{e \exp }$ in this case is

$B_{e \exp } \approx \int_{-v_{\mathrm{e}} / 2}^{+v_{\mathrm{e}} / 2}\left|D\left(v_{\exp }-v\right)\right|^{2} \mathrm{~d} v$.

When the filter frequency $v_{\mathrm{F}}=v_{\exp }+\varepsilon$, the frequency response is given by Eq. (A.2). The bandwidth noticed $B_{e \varepsilon}$ becomes
Close to each maximum signal peak, the NMV method refines the spectral peak without keeping up the amplitudes. These different points are illustrated in Fig. 3a which shows the NMV estimate of the same simulated signal as in Fig. 2. We clearly see the refining of the sinusoid peak compared with the MV estimation in Fig. 2a, but, in the broad band, some differences with the true PSD too. Fig. $3 \mathrm{~b}$ shows the variations of the bandwidth versus frequency. We can observe an increase just around the maximum of the sinusoid frequency. These

$B_{e \varepsilon}=\int_{-v_{\mathrm{e}} / 2}^{+v_{\mathrm{e}} / 2}\left|\frac{D\left(v_{\exp }+\varepsilon-v\right)-D\left(v_{\exp }-v\right)\left(1-\left(\pi M t_{\mathrm{e}}\right)^{2} \varepsilon^{2} / 6\right)^{2}}{\left(\pi M t_{\mathrm{e}}\right)^{2} \varepsilon^{2} / 3}\right|^{2} \mathrm{~d} v$

When $\varepsilon$ tends to 0 , we can show that $B_{e \exp } \ll B_{e \varepsilon}$. This fundamental relation means that the bandwidth greatly increases close to the maximum of a peak. So, the amplitude ratio of the two estimators can be deduced:

$\frac{S_{\mathrm{NMV}}(v+\varepsilon)}{P_{\mathrm{MV}}(v+\varepsilon)} \ll \frac{S_{\mathrm{NMV}}(v)}{P_{\mathrm{MV}}(v)}$.

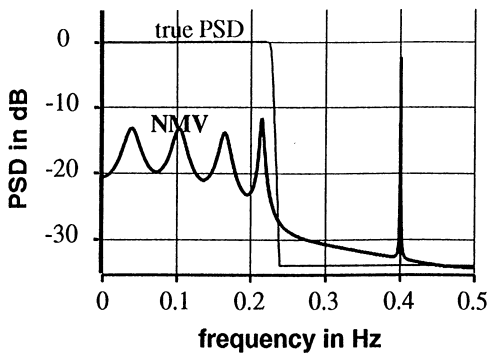

(a)

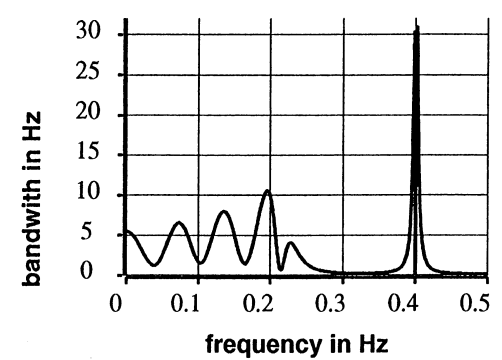

(b) figures show the way the frequency resolution is improved.

\section{A new estimator}

If we maintain the objective of defining a PSD estimator from a MV one, previous results must be

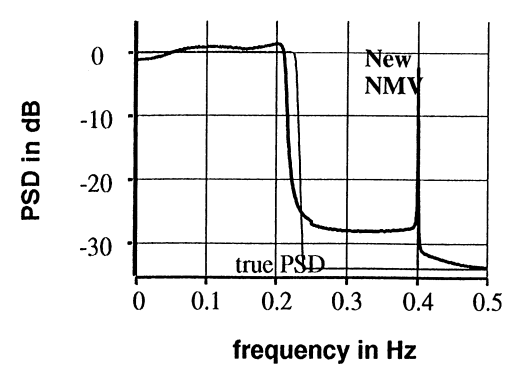

(c)

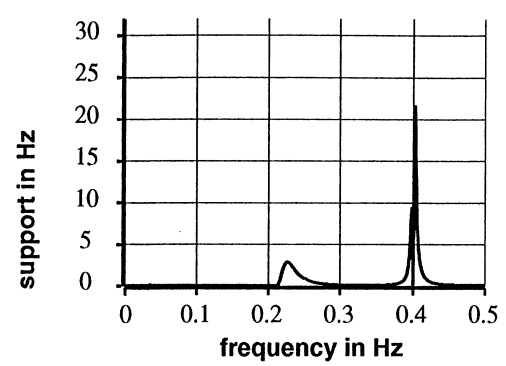

(d)

Fig. 3. The NMV DSP and the new one for the same signal as in Fig. 2. Filters order 12. (a) Overlaid NMV and true PSD, (b) $B_{\mathrm{e}}$ used for computing the NMV PSD, (c) overlaid new NMV and true PSD, (d) $B_{\bmod }$ used for computing the new NMV PSD. 
considered when Eq. (6) is applied. Due to the basic relation between a PSD and a power, we cannot pass round the notion of integral and of its integration support. But, due to the extreme particularity of the filter shape, we may not consider a classical bandwidth definition. The integration support must be adapted to the designed filter. In this section, we propose a definition of this support which leads us to define a new estimator. The performance of this estimator is then evaluated by a statistical study in order to compare it with MV and NMV ones.

\subsection{Definition}

The meaningful part of the frequency response at the filter frequency $v_{\mathrm{F}}$ lies in a band where the first constraint of the MV filter is actual, so we propose to only consider the lobe containing $v_{\mathrm{F}}$. The other lobes are meaningless, since they are in frequency bands where there is no high power. Let $\Delta B$ be the bandwidth of the lobe including $v_{\mathrm{F}}$. If $v$ is outside $\Delta B$, the affected part of the integral defined in (2) is equal to 0 , because either $S_{x}(v) \approx 0$ or $\left|A_{v_{\mathrm{F}}}(v)\right|^{2} \approx 0$. We can thus write

$$
P_{\mathrm{MV}}\left(v_{\mathrm{F}}\right) \approx \int_{\Delta B}\left|A_{v_{\mathrm{F}}}(v)\right|^{2} S_{x}(v) \mathrm{d} v
$$

Let us assume $S_{x}(v)$ is flat over $\Delta B$ range, i.e. $S_{x}(v) \approx S_{x}\left(v_{\mathrm{F}}\right)$. Therefore, Eq. (14) is written as

$$
P_{\mathrm{MV}}\left(v_{\mathrm{F}}\right) \approx S_{x}\left(v_{\mathrm{F}}\right) \int_{\Delta B}\left|A_{v_{\mathrm{F}}}(v)\right|^{2} \mathrm{~d} v .
$$

Substituting Eq. (5) into (15), we define a new estimator $S_{\text {mod }}\left(v_{\mathrm{F}}\right)$ as

$$
\begin{aligned}
& S_{\mathrm{mod}}\left(v_{\mathrm{F}}\right)=\frac{1}{E_{v_{\mathrm{F}}}^{\mathrm{H}} R_{X}^{-1} E_{v_{\mathrm{F}}} B_{\mathrm{mod}}} \\
& \text { where } B_{\mathrm{mod}} \approx \int_{\Delta B}\left|A_{v_{\mathrm{F}}}(v)\right|^{2} \mathrm{~d} v .
\end{aligned}
$$

The last integral must be evaluated. To be adapted to all signals, we propose a numerical definition

$$
B_{\text {mod }}=\Delta B\left|A_{v_{\mathrm{F}}}(v)\right|_{\max }^{2}
$$

where $\left|A_{v_{\mathrm{F}}}(v)\right|_{\max }^{2}$ is the maximum of the lobe containing $v_{\mathrm{F}}$.

The band $\Delta B=\left[v_{-}, v_{+}\right]$is precisely defined by $\left|A_{v_{\mathrm{F}}}\left(v_{-}\right)\right|^{2}=\left|A_{v_{\mathrm{F}}}\left(v_{+}\right)\right|^{2}=\left|A_{v_{\mathrm{F}}}(v)\right|_{\max }^{2} / 2$.

The chosen solution is the most simple to carry out. It should be possible to use the noise-equivalent bandwidth definition too, but only on the lobe including the filter frequency and without using Parseval's equality. In any case, a numerical solution cannot be avoided. The quantity $B_{\text {mod }}$ must be considered as a support of integration more than an estimation of a bandwidth.

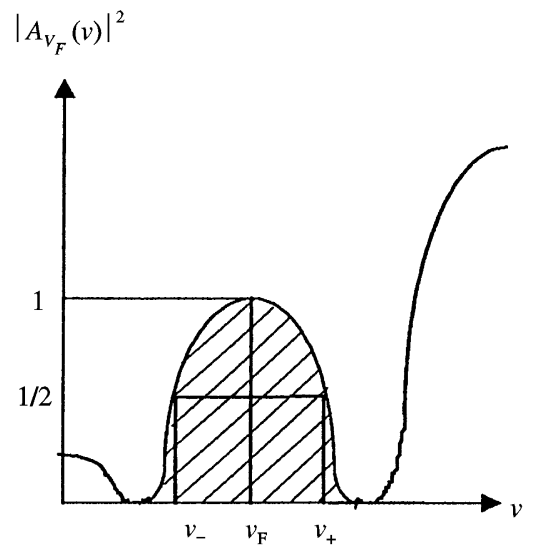

(a)

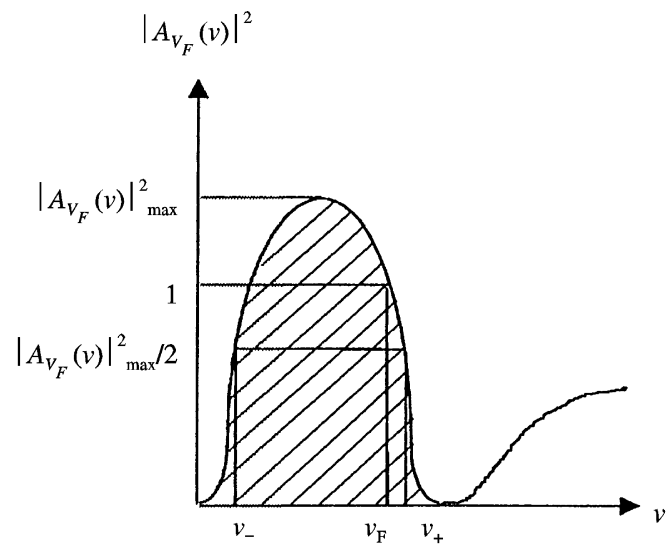

(b)

Fig. 4. Definition of the support integration $B_{\text {mod }}$, (a) $v_{\mathrm{F}}$ at a signal power peak, (b) $v_{\mathrm{F}}$ close to a signal power peak. 
This definition is illustrated in Fig. 4 in two cases. When the filter frequency is equal to the exponential frequency, that definition turns out to be exactly to the definition of the $-3 \mathrm{~dB}$ bandwidth because $v_{\mathrm{F}}$ is at the maximum of the focused lobe as shown in Fig. 4a. When the filter frequency is closer to a signal power peak the lobe is no longer centered at $v_{\mathrm{F}}$ and $\left|A_{v_{\mathrm{F}}}(v)\right|_{\max }^{2}$ is greater than 1 as shown in Fig. 4 b. So, the support $B_{\text {mod }}$ adapts to this configuration.

We test this estimator with the same signal as the one simulated in Section 2.4. Fig. 3c shows the PSD

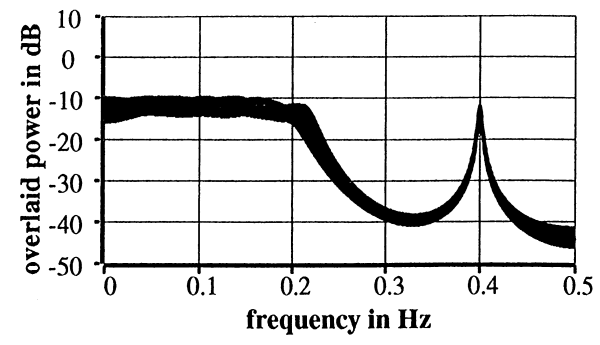

(a)

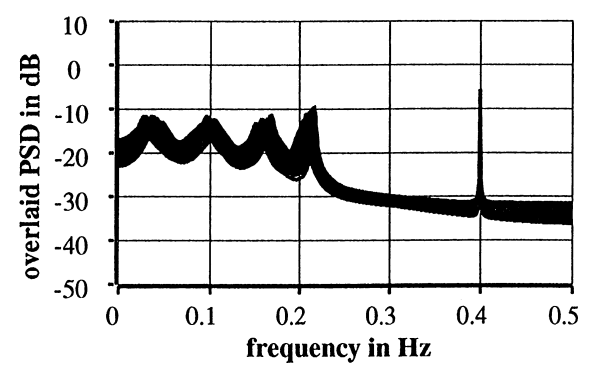

(b)

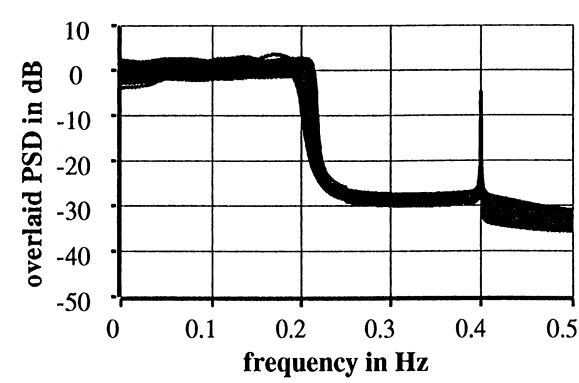

(c) obtained with the new estimator. We observe that the sinusoid peak is always refined but, in the broad band, the estimation is closed to the true PSD all along the band. Fig. 3d shows the support $B_{\bmod }$ vs. frequency as computed from (17). We observe a value increase when the filter frequency lies beside the sinusoid frequency or close to the broad band. Compared with the NMV estimator (Figs. 3a and 3b), fluctuations in the broad band are suppressed. At a sinusoid frequency, there is always a local minimum which implies a peak refining.

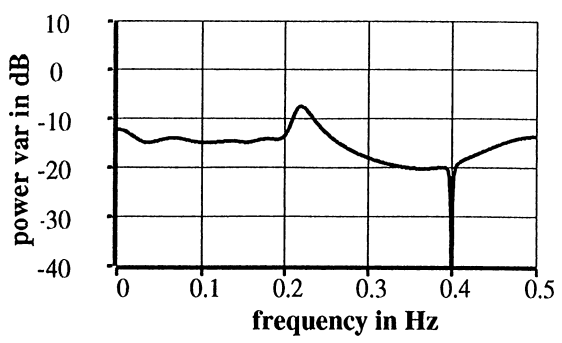

(d)

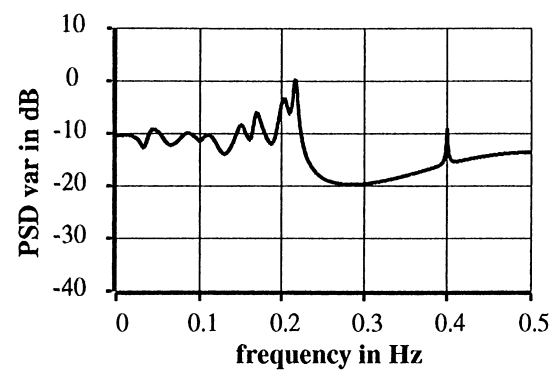

(e)

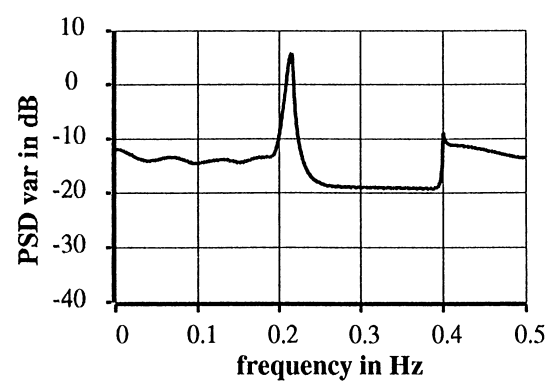

(f)

Fig. 5. Spectral estimation of hundred realizations of the signal used in Fig. 2. Filters order 12. (a) Overlaid MV power estimates, (b) overlaid NMV DSP estimates, (c) overlaid new NMV DSP estimates, (d) normalized variance of the MV power estimation, (e) normalized variance of the NMV DSP estimation, (f) normalized variance of the new NMV DSP estimation. 
Furthermore, at frequencies where the signal power is high, the support $B_{\text {mod }}$ lies in the correct order of magnitude as we might expect. For instance,

- at the sinusoid frequency, $B_{\text {mod }}=0.127 \mathrm{~Hz}$,

- at $v_{\mathrm{F}}=0.111 \mathrm{~Hz}$ within the broad band, $B_{\text {mod }}=0.068 \mathrm{~Hz}$.

Whereas with the NMV estimator, $B_{\mathrm{e}}$ equals to $1.626 \mathrm{~Hz}$ in the last case. This value is greater than 0.5 , which is the full frequency range of the signal! The filter shape and, more particularly, the value of the maximum which is no more equal to 1 , induces that unrealistic number which has really no meaning.

It is important to notice that the support $B_{\bmod }$, such as we defined it, cannot be considered as an estimation of the frequency resolution. It also has no relation with the sampling frequency or the signal frequency range. If $B_{\text {mod }}$ is large, it does not mean that the frequency resolution is small, but more often, that around this frequency the signal has no energy. This explains why $B_{\bmod }$ at the sinusoid frequency is relatively large, whereas if we measure the $-3 \mathrm{~dB}$ bandwidth of Capon's peak (Fig. 2a), we get $0.008 \mathrm{~Hz}$.

\subsection{Statistical study}

In order to compare the different MV estimators, hundred realizations of always the same signal as in Section 3.4 were simulated. Figs. $5 \mathrm{a}-\mathrm{c}$ show the overlaid estimations of the MV power, the NMV DSP and the new NMV DSP. The obtained biases are identical to those presented in Fig. 2a for MV, Fig. $3 \mathrm{a}$ for NMV and Fig. 3c for the new NMV. The normalized variances of these estimates are displayed in Figs. 5d-f. These simulations corroborate the previously described behavior. Compared with the MV estimator, the peak refining of the NMV estimator is also preserved in the new one. With regard to the estimate level in the broad band, the MV estimator has a bias of $-10 \mathrm{~dB}$ with respect to the true level. The NMV estimate is even lower whereas the new NMV one tracks correctly the true amplitude. The variances of the MV and new NMV estimators are nearly the same and lower than the NMV one except at the exponential frequency and close to the broad band.

\section{Conclusion}

This paper showed the importance of the filtering interpretation of MV method. We saw how the constraints influence the filter design. Consequently, we deduced that MV filters are not always narrow-band filters. This was not previously noticed.

Our study explained how the normalization introduced by Lagunas can produce not only interesting results but some artifacts too. The amplitude variations are unrealistically enhanced, leading to a strongly biased PSD estimation. We can state that the NMV method is more adapted to narrow spectral band signals and that the frequency resolution is improved compared with the MV estimator. But the NMV estimator is not adapted to track the amplitude and to ensure a good PSD estimation. In contrast, the new estimator we propose in this paper increases the frequency resolution compared with the MV method, sometimes less efficiently than the NMV method, but always preserves the PSD with lower bias and variance for any kind of signals even for mixed spectrum.

It relies on a scaling more adapted to Capon's filter behavior. Note that computation is a little harder and more time-consuming than Lagunas' method given that Parseval's equality could no longer be used. It is not possible to deduce the frequency resolution from this scaling factor and, at the moment, the only way to get a value of the frequency resolution is to measure it directly from the estimated power, as Lacoss did with a noised single sine wave [7].

\section{Appendix A}

In order to understand the effect of the smoothing factor in Eq. (11), this term is approximated at order 2

$$
\begin{aligned}
& D\left(v_{\mathrm{F}}-v_{\mathrm{exp}}\right) \approx 1-\frac{\left(\pi M t_{\mathrm{e}}\right)^{2}\left(v_{\mathrm{F}}-v_{\mathrm{exp}}\right)^{2}}{6} \\
& \text { and } D\left(v_{\mathrm{F}}-v_{\mathrm{exp}}\right)^{2} \approx 1-\frac{\left(\pi M t_{\mathrm{e}}\right)^{2}\left(v_{\mathrm{F}}-v_{\mathrm{exp}}\right)^{2}}{3}
\end{aligned}
$$


Substituting (A.1) into (11) gives the squared frequency response in case 2 :

$$
\begin{aligned}
& \left|A_{v_{\mathrm{F}}}(v)\right|^{2} \approx \\
& \left.\frac{D\left(v_{\mathrm{F}}-v\right)-D\left(v_{\exp }-v\right)\left(1-\left(\pi M t_{\mathrm{e}}\right)^{2}\left(v_{\mathrm{F}}-v_{\mathrm{exp}}\right)^{2} / 6\right)}{\left(\pi M t_{\mathrm{e}}\right)^{2}\left(v_{\mathrm{F}}-v_{\mathrm{exp}}\right)^{2} / 3}\right|^{2} \\
& C^{2} / \sigma^{2} \gg 1, \quad Q \approx 1 / M, \quad v_{\mathrm{exp}}-v_{\mathrm{F}} \rightarrow 0 .
\end{aligned}
$$

A stronger approximation can point out the property of this filter. In the frequency range close to $v_{\mathrm{F}}$ and $v_{\exp }$, we can limit all Dirichlet's functions to second order given that $\left(v_{\exp }-v_{\mathrm{F}}\right) \rightarrow 0$, $\left(v_{\exp }-v\right) \rightarrow 0$ and $\left(v_{\mathrm{F}}-v\right) \rightarrow 0$. So, Eq. (11) becomes

$$
\begin{aligned}
& \left|A_{v_{\mathrm{F}}}(v)\right|^{2} \approx \frac{1}{4}\left(1+\left(\frac{v_{\exp }+v_{\mathrm{F}}-2 v}{v_{\mathrm{exp}}-v_{\mathrm{F}}}\right)\right)^{2}, \\
& C^{2} / \sigma^{2} \gg 1, \\
& Q \approx 1 / M \\
& v_{\exp }-v_{\mathrm{F}} \rightarrow 0, v_{\exp }-v \rightarrow 0, v_{\mathrm{F}}-v \rightarrow 0 .
\end{aligned}
$$

This strong approximation is correct because the two MV constraints (1) and (2) are always respected. See Fig. 1e.

\section{References}

[1] M. Basseville, P. Flandrin, N. Martin (Eds.), Signaux non stationnaires. Analyse temps-fréquence et segmentation, Trait. Signal (Suppl.) 9(1) (1992).
[2] J. Capon, High resolution frequency wavenumber spectrum analysis, Proc. IEEE 57 (1969) 1408-1418.

[3] J. Capon, N.R. Goodman, Probability distributions for estimates of the frequency-wavenumber spectrum, Proc. IEEE 58 (1970) 1785-1786.

[4] G.H. Golub, C.F. Van Loan, Matrix Computations, The Johns Hopkins University Press, Baltimore, MD, 1985.

[5] E.E. Ioannidis, On the behavior of a Capon-type spectral density estimator, Ann. Stat. 22 (4) (1994) 2089-2114.

[6] S.M. Kay, Modern Spectral Estimation, Prentice-Hall Theory and Applications, Englewood Cliffs, NJ, 1988.

[7] R.T. Lacoss, Data adaptive spectral analysis methods, Geophysics 36 (4) (August 1971) 661-675.

[8] M.A. Lagunas, M.E. Santamaria, A. Gasull, A. Moreno, Maximum likelihood filters in spectral estimation problems, Signal Processing 10 (1) (January 1986) 19-34.

[9] S.L. Marple Jr., in: A.V. Oppenheim (Ed.), Digital Spectral Analysis With Applications, Prentice-Hall Signal Processing Series, Englewood Cliffs, NJ, 1987.

[10] J. Mars, N. Martin, J.L. Lacoume, M. Dubesset, Analysis of signal over short time windows, Signal Processing 26 (February 1992) 147-159.

[11] N. Martin, J. Mars, J. Martin, C. Chorier, A Capon's timeoctave representation, Application in room acoustics, IEEE Trans. Signal Process. 43 (8) (August 1995) 1842-1854.

[12] V. Pierson, N. Martin, Comparison of shape descriptors for parameters extraction of a time-frequency image, Seventh SP Workshop on Statistical Signal \& Array Processing, Québec, Canada, June 26-29 1994, pp. 259-262.

[13] V.F. Pisarenko, On the estimation of spectra by means of non-linear functions of the covariance matrix, Geophys. J. Roy. Astronom. Soc. 28 (1972) 511-531.

[14] P.J. Sherman, On the family of ML spectral estimates for mixed spectrum identification, IEEE Trans. Signal Process. 39 (3) (March 1991).

[15] T. Subba Rao, M.M. Gabr, The estimation of the spectrum, inverse spectrum and inverse autocovariances of a stationary time series, J. Time S. Anal. 10 (1989) 183-202. 